\title{
Butyryl-CoA Dehydrogenase Deficiency
}

National Cancer Institute

\section{Source}

National Cancer Institute. Butyryl-CoA Dehydrogenase Deficiency. NCI Thesaurus. Code C98863.

A rare autosomal recessive inherited disorder caused by mutations in the ACADSB gene. It is characterized by deficiency of the enzyme 2-methylbutyryl-CoA dehydrogenase which is involved in the metabolism of isoleucine. Signs and symptoms include vomiting, lethargy, irritable mood, and developmental delays. It may also lead to breathing difficulties, seizures, and coma. 